Review Article

\title{
Concept of Spectrum Disorder in Psychiatry
}

\author{
Prerna Kukreti', Prerak Kumar ${ }^{2}$, Harita Mathur ${ }^{3}$, Saurabh ${ }^{4}$, Dinesh Kataria ${ }^{5}$
}

${ }^{1}$ Associate Professor, ${ }^{2}$ Senior Resident, ${ }^{3,4}$ Junior Resident, ${ }^{5}$ Director Professor and Head, Department of Psychiatry and Drug Deaddiction Centre, Lady Hardinge Medical College and S. S. K. Hospital, New Delhi, India.

DOI: https://doi.org/10.24321/2581.5822.201913

\section{I $\quad \mathbf{N} \quad \mathbf{F} \quad \mathbf{O}$}

\section{Corresponding Author:}

Prerna Kukreti, Department of Psychiatry and Drug Deaddiction Centre, Lady Hardinge Medical College and S. S. K. Hospital, New Delhi, India. E-mail Id:

dearfrien@gmail.com

Orcid Id:

https://orcid.org/0000-0001-9535-8109

How to cite this article:

Kukreti P, Kumar P, Mathur H, Saurabh, Kataria

D. Concept of Spectrum Disorder in Psychiatry. J

Adv Res Psychol Psychother 2019; 2(3\&4): 29-34.

Date of Submission: 2020-01-24

Date of Acceptance: 2020-03-14

\section{$\begin{array}{llllllll}\mathbf{A} & \mathbf{B} & \mathbf{S} & \mathbf{T} & \mathbf{R} & \mathbf{A} & \mathbf{C} & \mathbf{T}\end{array}$}

Classification of mental disorders is a great tool to communicate amongst researchers, clinicians, epidemiologist as well as policy makers. Psychiatry nosology has been evolving with the advancement of scientific knowledge. Many approaches are used to classify the disorders ranging from categorical to dimensional to hybrid approaches. Categorical approach is helpful in delineating the prototype of disorder but spectrum concept helps in understanding the subthreshold symptoms, heterogeneity in presentation of same disorder and comorbidity across disorders. No system of nosology can be perfect until precise and accurate cause of mental illness will be known, but spectrum approach may hold the promise for being more accurate, heuristic and holistic.

Keywords: Categorical Approach, Spectrum Approach, Nosology, Mental Illness Classification, Subthreshold Symptom

\section{Introduction}

A spectrum disorder in psychiatry refers to a range of linked conditions, sometimes also extending to include singular symptoms and traits. The different subgroups of a spectrum either co-occur more than often or have a similar phenomenology or share common neurobiological underpinnings. Spectrum approach suggests for presence of "not a unitary disorder but rather a syndrome composed of subgroups". The spectrum may represent a range of severity, comprising relatively "severe" mental disorders through to relatively "mild and nonclinical deficits".

In some cases, a spectrum approach joins together conditions that were previously considered separate for example in autism spectrum and obsessive spectrum disorders, in other cases, it can suggest a range of conditions for an entity initially considered as a single disorder for example in bipolar spectrum disorder. It not just encapsulates pathological conditions, but also envisages some of the traits in general population to subthreshold symptoms to core clinical conditions to atypical symptoms. It offers a panoramic view of the mental health conditions over the prototypical yes/ no answers of myopic categorical classification and helps clinicians to personalize the diagnosis.

\section{Origin of the Concept}

The concept of spectrum traces back to its origin in physics for an apparent qualitative distinction arising from a quantitative continuum (i.e., a series of colors formed when a beam of white light is dispersed by a prism so that their parts are arranged in the order of their wavelengths). ${ }^{2}$ In psychiatry, this analogy has been used to demarcate a group of disorders qualitatively distinct in appearance (e.g., depression and anxiety disorder) but believed to be stemming from a common pathogenetic point of view. ${ }^{3}$ For different investigators, the hypothetical common pathogenetic link has been of a different nature: familialgenetic (i.e., schizophrenia spectrum); ${ }^{4}$ response to a class of pharmacologic treatments (i.e., depressive spectrum); ${ }^{5}$ common underlying psychopathologic traits (i.e., dissociative spectrum); ${ }^{6}$ the combination of some of the foregoing validating strategies (i.e., bipolar spectrum); ${ }^{7}$ range of 
more than frequent comorbid conditions with common psychopathology (i.e. in obsessive compulsive spectrum. ${ }^{8}$

\section{Historical Evolution in Nosology: Shift from Spectrum to Categorical Models}

The wisdom of spectrum disorder in words may appear new, but conceptual underpinnings can date back to more than a century. Reflections can be seen in the early works of Bleuler, where he described odd eccentric relatives of schizophrenia patients without overt psychotic symptoms as "Iatent schizophrenia", Kretschemr described temperamental predisposition to schizophrenia or "schizoid personality", Rado coined the term "schizotype" which further laid the foundation for seminal work of Meehl giving rise to concept of "schizotaxia". ${ }^{4}$

Historically, these all authors suggested for a spectrumbased approach, but, the famous project by Cooper et al. ${ }^{9}$ showed that the huge epidemiological discrepancy across continents in mental disorders was more due to the diagnostic differences than real differences in prevalence. Thus, the need of operationalization and standardization paved way for birth of categorical classificatory systems, International Classification of Diseases (ICD) and Diagnostic Statistical Manual (DSM).

\section{Need of Shift from Categorical Concept to Spectrum Concepts}

These categorical systems of classification undoubtedly offered certain discrete advantages:

- It made the communication uniformly standardized. Clinicians could easily communicate to the patient, other clinicians and third parties like insurance companies or epidemiologist.

- It was more in alliance with the medical model of disease across other disciplines increasing acceptability.

However, the categorical models did not take into account the following concerns:

- $\quad$ The validity of the categorical approach has been questioned by the vast heterogeneity of the diagnosis - the "x symptoms out of $y$ " approach leading to numerous different clinical combinations with little in common apart from the diagnosis. ${ }^{4}$

- Contradictory evidence emerged form long-term longitudinal studies raising question on long term validity of certain categorical diagnosis. For example, some longitudinal studies demonstrated the existence of cases Beginning as Bipolar Disorder (BPD) and later turning into Schizophrenia (SZ), as well as, vice versa. ${ }^{10,11}$ At 5-years follow up, $63.6 \%$ of 55 subjects, aged $12-20$ years, consecutively hospitalized for a manic or mixed episode still had a diagnosis of BPD; $14.5 \%$ changed life-time diagnosis for Schizoaffective (SAD) disorder and $20 \%$ for SZ. ${ }^{12}$

- Clinicians often felt these diagnostic systems as being unreflective of theory and silent to idealized description of a patient or individualized treatment. Patients with same diagnosis used to vary widely in symptoms, severity, course and outcome.

- Most of the epidemiological studies showed high comorbidity amongst several disorders, raising a concern that were these real comorbidities or mere co-existence of real disorders lying on a spectrum together. ${ }^{13-15}$

- The heterogeneity of patients receiving a particular diagnosis became substantial; making etiological and neuropathological research's application limited. Tendency to view research findings through primitive syndromic lens of categorical classification was actually seen as impediment to biological research process. ${ }^{16}$

- $\quad$ Research proved that many of these mental disorders were in fact constructed from several discrete dimensions such as a cognitive dimension, an impulsivity dimension, and dimensions of positive and negative symptoms in schizophrenia spectrum disorders. ${ }^{4}$

- $\quad$ Large numbers of relatively common genetic risk alleles identified were pleiotropic, according well with the fuzzy boundaries of diagnostic labels. It again pushed the hypothesis forward that possibly we are dealing with traits that are best conceived of as continuous or dimensional rather than categorical at both the phenotypic and genotypic levels. ${ }^{16}$

- Many of the patients with subthreshold symptoms could not find due place in diagnostic system, despite it being a significant cause of distress and significant predictor of future morbidity. ${ }^{17}$

- Results from Analogue samples study design (Outside of medical settings, the study of subthreshold symptoms is called analogue research, which uses, for example, nonclinically depressed university students as analogues for "real" clinical depression) showed that some of the disorders like depression were lying on a continuum in which symptoms only differed in intensity as compared to analogue samples, further advocating for presence of a spectrum approach than clear zone of rarities between disorders as demarcated in categorical systems. ${ }^{17}$

- $\quad$ Since the categorical dichotomy had no space for individual variations in symptoms, or non-core criteria symptoms; it produced a pseudo picture of many clients being clubbed into "Not otherwise specified (NOS) categories". ${ }^{17}$

It will not be wrong to say that clinical utility and ease of usage in clinics of current categorical system has obscured the concern about lack of validity of categorical systems of classification. ${ }^{16}$ Unfortunately deification of categorical system of classification led to reification of psychiatric diagnosis. Thus, it calls for a much needed, paradigm shift 
to start working on lines of spectrum concept of disorders again.

\section{Spectrum Related Concepts and Definitions}

Before elaborating on the spectrum concept, it is important to clarify few terms for academic discussion, however, during the text, they may be found to be used interchangeably. ${ }^{17}$

Continuum: It refers to a continuous panorama of traits seen as a continuous gradient from normal to abnormal. Unlike spectrum, it does not consider any significant discontinuity or clear zones of separation between related conditions (Figure 1).

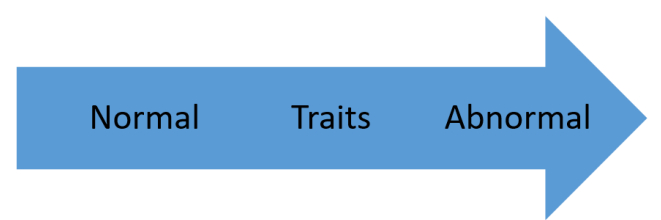

\section{Figure I.Continum concept presumes no zone of demarcation}

Spectrum: Refers to a range of qualitatively distinct conditions united together by a common underlying phenomenology or neurobiology (Figure 2).

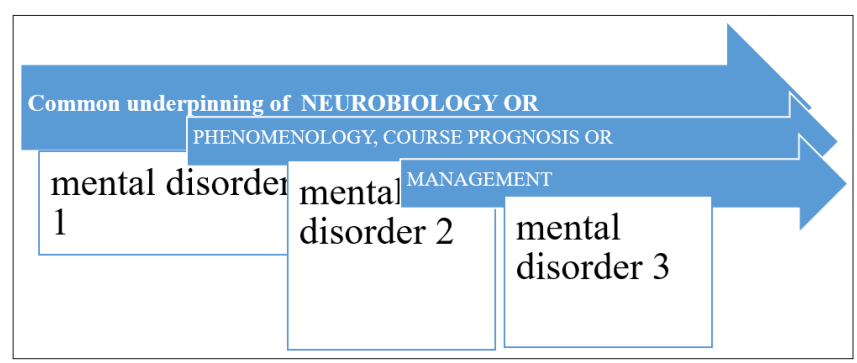

Figure 2.Spectrum concept: Links together disorders on basis of few common underpinnings

Dimensions: The qualitative distinction present within a disorder or spectrum of disorders or continuum can be measured through domains called 'dimensions', which can vary in terms of either a behavior or phenomenology or severity.

Threshold: Refers to the number of symptoms and their duration, at or above which a mental disorder is said to be present as per ICD or DSM.

Subthreshold: Refers to range of 'low grade symptoms' which may be the reason of consultation or distress but are below the threshold of symptoms mentioned in ICD or DSM.

Criterion Symptoms: They are mentioned as core, cardinal or primary symptoms of a particular disorder in ICD or DSM.

Non-Criterion Symptoms: It refers to certain symptoms known to be present in a disorder, but have been mentioned as atypical symptoms in ICD or DSM and till ICD-10 and DSM IVTR had found place mostly in NOS categories.
Interestingly, spectrum concept doesn't discard categorical classification approach, but it subsumes it and expands beyond it also to traits, lifestyle or behavioral patterns, personality characteristics as well as a range of linked conditions ranging from abnormal experiences found in general population (e.g. hallucinations experienced by general population ${ }^{4}$ ) to pathological personality traits, to threshold symptoms to subthreshold as well as atypical symptoms (Figure 3).

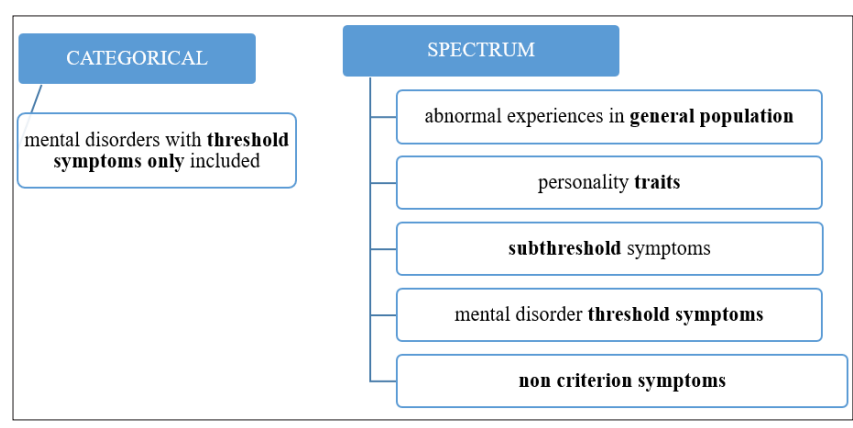

Figure 3.Difference in approach of categorical and spectrum concept

Further, Spectrum disorders can be rated on several dimensions, which can vary in terms of specific domains of psychopathology, severity of illness, level of functioning or certain specifiers connoting to particular phase or course of illness giving a more holistic and individualized view about an individual suffering from any disorder (Figure 4).

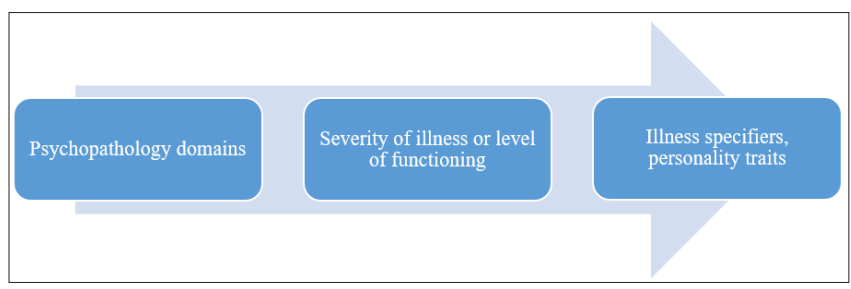

Figure 4.Spectrum disorder can be graded on any of these dimensions

\section{Classificatory Systems Based on Spectrum Concept Alone}

\section{Research Domain Criteria}

Despite the euphemism of past few decades of progress in biological research, field of psychiatry is yet to witness the translation of explosion of knowledge in the clinical or diagnostic approaches. However, National Institute of Mental Health's (NIMH) Research Domain Criteria (RDoC) project appears as a silver lining in the dark clouds of so far prevalent theoretical approach. ${ }^{16}$

Basic working frame of RDoC: It is a 'multidimensional system' ranging from normal to abnormal. It has been conceptualized as a matrix along "four dimensions": Neurodevelopmental, environmental, behavioral and domains of functioning. Domains of functioning have been 
further divided into five dimensions named "constructs" namely negative valence (systems that enable response to aversive stimuli or contexts like threat, loss, aggression due to frustration), positive valence (systems that mediate reward related activity e.g. reward responsiveness), cognitive systems (such as attention, perception, memory), social processes (such as affiliation, facial expression) and arousal and modulatory systems (such as circadian rhythms). Further seven "unit of analysis" have been defined to measure the neurobiological aspect of these dimensions, namely, genes, molecules, cells, neural-circuit activity, physiology, behavior and self-reports. ${ }^{16}$

It will classify behavior and will try to understand etiology rather than just putting the label of a single diagnosis. Intent of RDoC is to increase attention to neurodevelopmental and environmental effects to free researchers from having to use description-oriented categories alone and to encourage them on focusing more on basic mechanisms more relevant to etiology (Figure 5). However, limitations of such purely biological systems of classification is that they are currently only ready for research work and will take long to translate into working tools of clinical utility. ${ }^{16}$

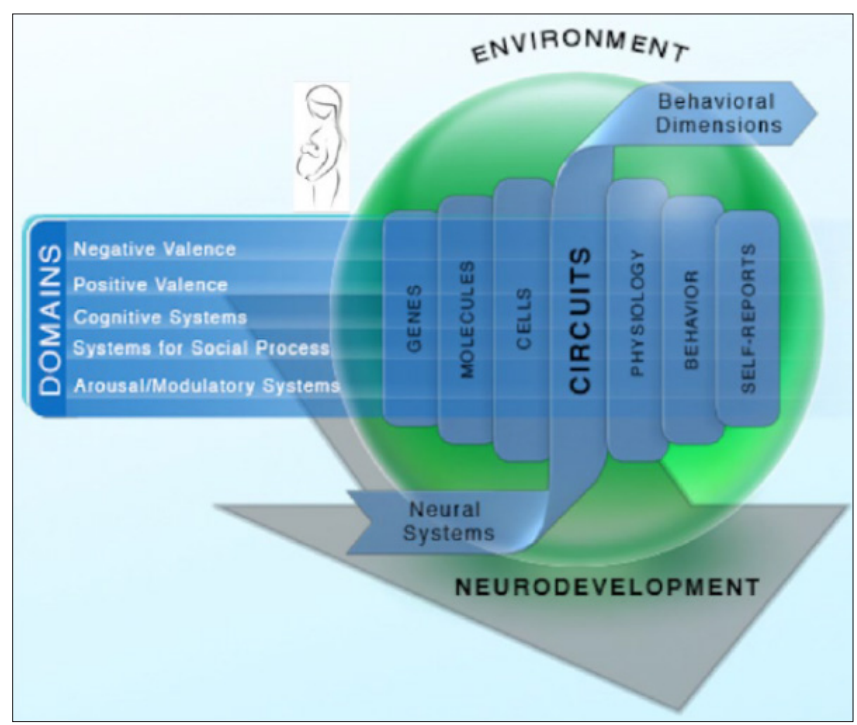

Figure 5.Multidimensional approach of RDoC project Horizons Meeting: Classificatory Systems Based on Categorical and Spectrum Concept

\section{Diagnostic Statistical Manual 5 (DSM-5)}

DSM 5 has innovated to supplement its categorical system by introducing dimensionality in following ways:

1. Nosological System Incorporating Spectrum Disorders: DSM 5 has legitimized this thought of spectrum concept and has incorporated several formal spectrum disorders which combine highly related disorders such as:

a) Autism Spectrum Disorders: It merges all the disorders with persistent deficits in social communication and restricted/repetitive behavior/interests. It incorporates previous diagnosis of autism, Asperger, or pervasive developmental disorder NOS.

b) Schizophrenia Spectrum and Other Psychotic Disorders: It incorporates schizophrenia, other psychotic disorders and schizotypal personality disorder.

c) Bipolar Related Disorders: It has been separated from depressive disorders in DSM 5 and placed between schizophrenia spectrum disorder and depressive disorders in recognition of their place in nosology as a bridge between two diagnostic entities. It now includes bipolar I and II disorder, cyclothymic disorder as well as substance/ medication induced bipolar and related disorder.

d) Obsessive Compulsive and Related Disorders: It has now been separated from other anxiety disorders and includes obsessive compulsive disorder, body dysmorphic disorder, hoarding disorder, trichotillomania, excoriation disorder and substance/ medication induced obsessive compulsive and related disorder. They all are related to each other in terms of diagnostic validators, frequent comorbidity, and treatment options.

e) Trauma and Stressor Related Disorders: after separation from anxiety disorders, it now constitutes a spectrum in which exposure to a traumatic or stressful event is listed explicitly as a diagnostic criteria and patients should have an hedonic and dysphoric symptoms as prominent clinical presentation rather than anxiety or fear. It includes reactive attachment disorder, disinhibited social engagement disorder, post-traumatic stress disorder, acute stress disorder and adjustment disorder.

f) Somatic Symptom and Related Disorders: This spectrum shares the common feature of prominence of a somatic symptom plus abnormal thought, feeling, behavior in response to it, associated with significant distress and impairment rather than absence of a medical cause of same. It includes somatic symptom disorder, illness anxiety disorder, conversion disorder, factitious disorder and psychological factors affecting other medical conditions.

g) Substance Related and Addictive Disorders: It includes a cluster of cognitive, behavioral, and physiological symptoms in an individual consuming substance despite significant problems related to it. It now subsumes the category of abuse and dependence into rubric of 'substance use disorder' with grading of severity.

2. Use of Severity Rating as a Dimensional Approach: DSM 5 has included severity rating across all disorders by rating of psychopathology through use of some online assessment measures provided as rating scales and rating of functioning or impairment through use of WHO DAS (disability assessment scale). They have also 
included "cross cutting symptom measure" a broadband assessment measuring common psychiatry symptoms across diagnostic boundaries. Use of such measures is hoped to add surplus information helpful in aiding diagnosis, case monitoring and treatment planning. ${ }^{18}$

3. Use of Specifiers as a Dimensional Approach: DSM 5 has added a separate dimension of specifiers added across different entities concerning course or phenomenology to enrich the diagnosis. For example, specifiers like 'anxious distress; and "mixed features" have been shown to increase suicide risk and pretend a more complicated treatment regime. ${ }^{18}$

4. Dimensional Model for Personality Assessment: In addition to previous system, DSM 5 has added an alternative dimensional model of personality assessment characterized by impairment in personality functioning and pathological personality traits. This approach can also include a diagnosis of Personality Disorder-Trait Specified (PD-TS) that can be made when dimensional criteria for personality disorder are present but not for a specific disorder.

\section{Promises that Spectrum Disorder Concept Can Hold in Mental Health}

1. Mental health or mental disorder if envisaged on a spectrum can help in reducing the problem of just labelling the individuals without personalizing specifics of their problem, which can aid clinicians in formulating better management plans.

2. It can increase use of measurement-based approach for clinicians, facilitating gauging not just deteriorating but also improvement.

3. It can reduce the problem of undue increase in comorbidities to some extent.

4. Most patients can escape the problem of being labelled NOS and can get a valid diagnosis to aid management.

5. It can help researchers to work on the common neurobiological underpinnings of linked disorders from etiological perspective.

6. It can be of great value for epidemiologist to be told about the spectrum inclusive of subthreshold conditions also which are important cause of disability from public health perspective.

\section{Challenges for Application of Spectrum Disorder Concept}

1. Concerns about threshold: Determining appropriate threshold or cut offs for dimensions is critical in terms of determining true psychopathology. If the bar is set too low, there is a danger of pathologizing normal behavior. If set too high, those who need treatment may be excluded and denied services. For example, those who formerly might have received diagnosis of mild to moderate Asperger or substance abuse now may no longer classify for a diagnosis. ${ }^{18}$
2. Social Ramifications: Lumping mild to moderate cases under one rubric can have unintended social ramifications, especially for people on benign end of the spectrum. For example, a college student who could formerly receive diagnosis of alcohol abuse will now be clubbed with others being considered dependent under single rubric of 'substance use disorder' raising concerns about perceived stigma and consequent help seeking. ${ }^{18}$

3. Burden on Clinicians: Some of the busy clinicians may find it as an added burden to do extra assessment on dimensional system in due to time constrains.

Spectrum concept and dimensionality may be an important innovation in development of the new and innovative classificatory systems, but there are significant challenges ahead in calibrating these dimensions, refining measures and considering social consequences.

\section{Conclusion}

No system of nosology can be perfect until precise and accurate cause of mental illness will be known. But the walk down the lanes of spectrum approach can be more accurate, heuristic and holistic. Although, how all stakeholders will benefit from or adapt to this change future application can only tell but clinicians and clinical researchers should welcome it.

\section{Conflicts of Interest: None}

\section{References}

1. A video introduction to RDoC (Research Domain Criteria): A spectrum or dimensional approach to understanding and classifying mental disorders from the U.S. National Institute of Mental Health, 2013. Available from: www. nimh.nih.gov > news > media > 2013 > introduction-tordoc. Last accessed on 23 Feb 2020.

2. Halliday D, Resnick R, Walker J. Fundamentals of physics. $5^{\text {th }}$ Edition. John Wiley \& Sons, New York. 1997.

3. Winokur G. Depression spectrum disease: description and family study. Compr Psychiatry 1972; 13: 3-8.

4. Ritsner M. Handbook of schizophrenia spectrum disorders, Vol I: Conceptual issues and noslogical advances. $1^{\text {st }}$ edition. Springer, London. 2011.

5. Hudson JI, Pope HG. Affective spectrum disorder: does antidepressant response identify a family of disorders with a common pathophysiology? Am J Psychiatry 1991; 148: 548.

6. Shoda H. Splitting phenomena from a viewpoint of experiencing time: Spectrum from multiple personality and hysteria to borderline personality disorder. Psychopathology 1993; 26: 240-254.

7. Akiskal HS, Bourgeois ML, Angst J et al. Re-evaluating the prevalence of and diagnostic composition within the broad clinical spectrum of bipolar disorders. J Affect 
Disord 2000; 59(Suppl 1): 5s-30s.

8. Hollander E. Obsessive compulsive spectrum disorders: an overview. Psychiatric Annals 1993; 23: 355-358.

9. Cooper JE, Kendell RE, Gurland BJ et al. Psychiatric diagnosis in New York and London. Maudsley Monograph 20. Oxford University Press, Oxford. 1972.

10. Angst J. Zur Ätiologie und Nosologie endogener depressiver Psychosen. Eine genetische, soziologische und klinische Studie (Ger). Springer, Berlin. 1966.

11. Sheldrick C, Jablensky A, Sartorius N et al. Schizophrenia succeeded by affective illness: catamnestic study and statistical enquiry. Psychol Med 1977; 7: 619-624.

12. Cohen D, Guilé JM, Brunelle J et al. Bipolar episodes in adolescents: diagnostic issues and follow-up in adulthood. Encephale 2009; 35(Suppl 6): S224-S230.

13. Brieger P. Comorbidity in bipolar affective disorder. In: Marneros A, Angst J, editors. Bipolar disorders: 100 years after manic depressive insanity. Kluwer Academic Publishers, Dordrecht (The Netherlands). 2000; 215-229.

14. Fyer AJ, Liebowitz MR, Klein DF. Treatment trials, comorbidity, and syndromal complexity. In: Maser JD, Cloninger $\mathrm{CR}$, editors. Comorbidity of mood and anxiety disorders. American Psychiatric Press, Washington, DC. 1990; 669-679.

15. Perugi G, Akiskal HS, Ramacciotti S et al. Depressive comorbidity of panic, social phobia, and obsessivecompulsive disorders re-examined: is there a bipolar Il connection? J Psychiatr Res 1999; 33: 53-61.

16. Owen MJ. New Approaches to Psychiatric Diagnostic Classification. Neuron 2014; 84: 564-571.

17. Maser JD, Patterson T. Spectrum and nosology: implications for DSM-V. Psychiatr Clin N Am 2002; 25: 855-885.

18. DSM-5 Conceptual changes: Innovations, limitations and clinical implications. TPC Journal. Available from: http://tpcjournal.nbcc.org/dsm-5-conceptual changesinnovations-limitations-and-clinical-implications. 